\title{
Design of Wireless Acceleration Sensor Nodes Used in Mechanical Equipment Status Monitoring
}

\author{
G. VamshiKishore, J. Suresh
}

Department of ECE, Jyothishmathi College of Engineering and Technology, India

\begin{tabular}{l} 
Article Info \\
\hline Article history: \\
Received Jan 9, 2016 \\
Revised Feb 13, 2017 \\
Accepted Feb 20, 2017 \\
\hline
\end{tabular}

\section{Keyword:}

Acceleration Sensor ARM7

Mechanical Equipment Sensor Nodes Wireless Sensor Network ZigBee

\begin{abstract}
Wireless Sensor Network is a new integrated technology, which combines sensor technology, wireless communication technology, Micro-ElectroMechanical Systems technology and Digital electronic technology. Now, it has been widely used in military, industrial control, environmental monitoring, transportation, and medical. Along with the research evolution on Wireless Sensor Network, its application fields are gradually expanding. It is penetrating deep into each aspect of the human's production and life, and will be a new revolutionary technology. In this paper, a wireless acceleration node based on wireless single chip processor CC2430, an ARM7TDMI-S core based microcontroller called LPC2478 microprocessor and three-axis acceleration sensor ADXL330 is designed to monitor the mechanical system operation conditions and fault diagnosis with high efficiency. The performance and characteristics of CC2430, ADXL330 and LPC2478 are introduced compactly. The designations of hardware as well as software of wireless network node are introduced in details. The nodes perform steadily and efficiently in practical test, and it can be easily applied to other monitoring areas.
\end{abstract}

Copyright $@ 2017$ Institute of Advanced Engineering and Science. All rights reserved.

\section{Corresponding Author:}

G. VamshiKishore,

Department of ECE, Jyothishmathi College of Engineering and Technology, India.

Email: vamshi0463@gmail.com

\section{INTRODUCTION}

A Wireless Sensor Networks (WSNs) is a network consisting of spatially distributed self-organizing devices which use their sensors to monitor devices. Their sensors can monitor environmental conditions, such as mechanical system operation conditions, temperature, sound, pressure vibration. After data acquisition and information processing decision is made. WSNs consist of large number of sensor nodes which have the capability of computing, sensing and communicating. Compared to other embedded systems most sensor nodes are low-cost and resources-constrained and it can be deployed in a large scale [1]. Because most of the sensor nodes are battery powered, Energy efficiency is one of the most challenging issues. In additional, the software in deployed WSNs often needs to be changed, such as updating the system with new functionality, or fixing software bugs. Usually a large number of sensor nodes are spread over a large field which is usually unreachable and updating software manually is impossible. So the sensor nodes need remote reprogramming ability in order to add new functionality or fix software bugs. In view of the technical characteristics of wireless sensor networks, a new technical standard, the ZigBee technology [2], was proposed in 2002. It has obtained the widespread support after updating and improving. It has many desired properties, such as near distance, low complexity, the low powerless, the low date rate, the low cost. ZigBee technology as one kind of emerging wireless network technology is mainly used in the near distant wireless connection. It has its own agreement standard to communicate between several thousand small sensors. These sensors only need very little energy. They pass the data through the radio wave from a sensor to anther in the form of relay, therefore they communicate with an extreme by high efficiency wireless connection. 
WSNs were originally motivated by military applications for instance battlefield surveillance. Today, WSNs has obvious ascendancy over traditional wired sensors and become one of the hottest research directions of computer science in addition areas, including industrial control, environment monitoring, home automation, and traffic control. Wireless Sensor Network to monitoring mechanical system operation conditions and fault diagnosis has been significantly applied. We can monitor mechanical equipments by Wireless Sensor Network. It would help us to conveniently and quickly learn its operating conditions, and analyze the potential fault correctly, which will enhance the efficiency of equipment maintenance.

\section{SYSTEM DESIGN}

\subsection{Wireless Sensor Network Structure}

The sensor network system often includes sensor nodes, sink nodes, wireless bridge and management nodes, as shown in Figure 1. There are a large number of sensor nodes randomly deployed in the sensor field. They may form a network in a self-organized way. Sensor nodes located in the sensor field collect mechanical equipment status at the scene through acceleration sensor, which are sent to the Internet and finally to the task management centre after being digitally processed. Conversely, the task management centre can also send commands to sensor nodes through the wide area network and wireless LAN to monitor the mechanical equipment status. Currently, there are some technologies to achieve WSN, including ZigBee, WI-Fi, Bluetooth and other technologies. Of the above three technologies, we choose we choose ZigBee technology as the wireless communication approach which is the most widely used and has the lowest developing costs.

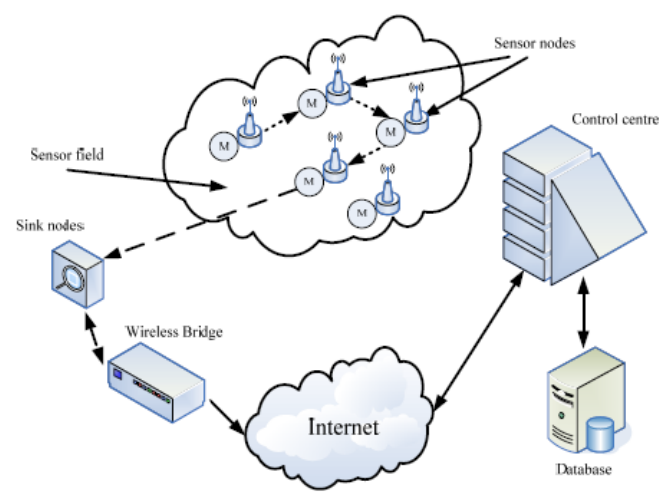

Figure 1. Sensor Network Architecture

\subsection{Hardware Design}

Wireless acceleration sensor nodes used inmechanical equipment status monitoring consists of two parts: sink node (coordinator) and router (or RFD). Coordinator is mainly responsible for building a wireless local area acceleration sensor network for packet routing and indirect news forwarding [3]. The task of the Router and RFD is to receive acquisition data command from coordinator and send the acceleration value of three directions to the coordinator periodically. The structure of wireless acceleration sensor nodes is shown in Figure 2. The CPU used in the coordinator is NXP Semiconductors LPC2478, which is designed to provide hand-held devices and general applications with low-power, and high-performance microcontroller solution. The LPC2478 microcontroller has $512 \mathrm{kB}$ of on-chip high-speed flash memory, which includes a special 128-bit wide memory interface and accelerator architecture that enables the CPU to execute sequential instructions from flash memory at the maximum $72 \mathrm{MHz}$ system clock rate. The LPC2478 can execute both 32-bit ARM and 16-bit Thumb instructions. The LPC2478 has the c haracteristics of real-time debug interfaces that include both JTAG and embedded trace. The LPC2478 offers outstanding features with an LCD controller, a 10/100 Ethernet Media Access Controller (MAC), a USB full-speed Device/Host/OTG Controller with $4 \mathrm{kB}$ of endpoint RAM, four UARTs, two Controller Area Network (CAN) channels, an SPI interface, two Synchronous Serial Ports (SSP), three I2C interfaces, and an I2S interface. These features make this device optimally suited for portable electronics and Point-of-Sale (POS) applications [4]. In additional, wireless module (ZigBee) is connected on the LPC2478 processor via a UART. Ethernet module is connected on the LPC2478 processor via SPI, which can sends data to the server of control center by 
Internet. The core components of the router (or RFD) are CC2430 and three-axis accelerometer ADXL330. The CC2430 includes a configurable resolution ADC ( 7 to 12 bits) with external eight-channel. With this, there is no need for an addition of AD conversion device in external system. At VS $=3 \mathrm{~V}$, the output of the ADXL330 typically varies 0V to $3 \mathrm{~V}$. Because the ADXL330 has provisions for band limiting the XOUT, YOUT, and ZOUT pins, the capacitors must be added at these pins to implement lowpass filtering for antialiasing and noise reduction. The XOUT, YOUT, and ZOUT pins of the ADXL330 are connected on the CC2430 via three channels ADC to get the three-axis acceleration.
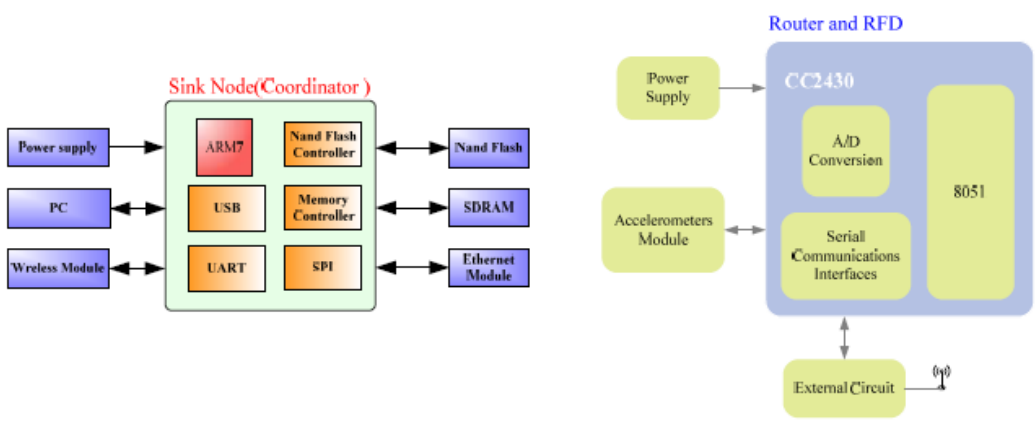

Figure 2. Block Diagram of Wireless Sensor Netwotk Node

\subsection{Wireless module}
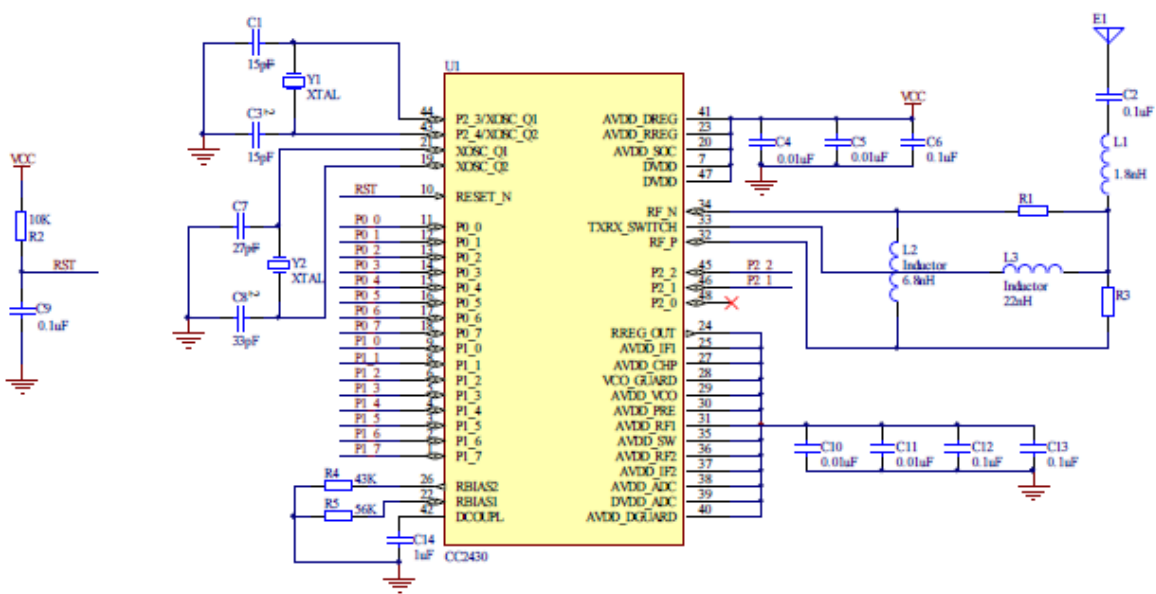

Figure 3. Wireless Module Circuit

The sensor nodes are responsible for collecting mechanical equipment status in sensor field and implement the timely transmission of data. In our project, the CC2430 [5] is choosing as ZigBee wireless module. The CC2430 is a true resolution to System-on-Chip (SoC) and highly suited for systems where ultra low power consumption is required. The CC2430 combines the excellent performance of the leading CC2420 $\mathrm{RF}$ transceiver with an high performance and low power 8051 microcontroller core, 32/64/128 KB flash memory, $8 \mathrm{~KB}$ RAM and many other powerful features. After power up, CC2430 does basic hardware initialization and determines whether to be configured as a network coordinator or terminal nodes according to the real situation. After that, sensor nodes can collect and send data. Figure 3 shows circuit of CC2430.

\subsection{Accelerometers module}

\subsubsection{Accelerometer measurement principle}


When the sensor remained relatively static, due to gravity, this acceleration sensor output an equal and opposite acceleration acting against the acceleration of gravity. The output signal of three-axis accelerometer depends on the angle of the three sensitive axes relative to gravity.

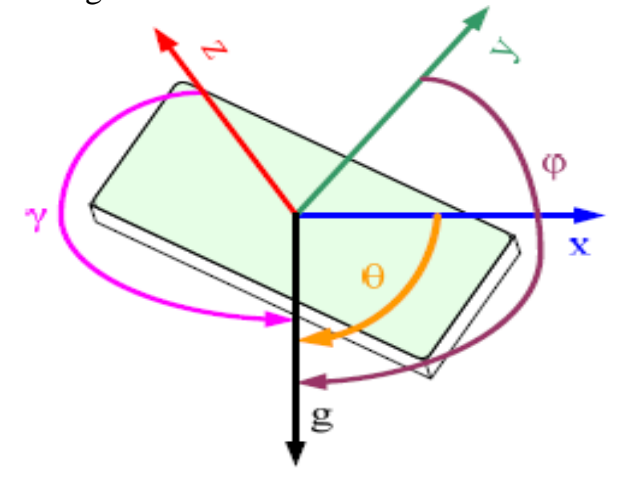

Figure 4. Using a Tri-axis Accelerometer with Three Axes for Measuring Tilt

Figure 4 shows $\mathrm{x}, \mathrm{y}$ and $\mathrm{z}$ accelerometer axes tilt relative to gravity. $\theta, \varphi$ and $\gamma$ represent the angles that the $\mathrm{x}, \mathrm{y}$ and $\mathrm{z}$ accelerometer maxes make with the fixed reference gravity. These angles can be calculated as the following:

$$
\left\{\begin{array}{l}
v_{x}=k g \cos \theta+v_{0} \\
v_{y}=k g \cos \varphi+v_{0}, \\
v_{z}=k g \cos \gamma+v_{0}
\end{array}\right.
$$

Where vx, vy and vz are the output voltage signal from the $\mathrm{x}, \mathrm{y}$, and $\mathrm{z}$ axes of accelerometer; $\mathrm{k}$ is the sensitivity of the accelerometer; $g$ is the gravity acceleration; when the acceleration is 0 , $v 0$ is the output voltage of accelerometer. Find the inverse of the above functions:

$$
\left\{\begin{array}{l}
\theta=\arccos \left(\frac{v_{x}-v_{0}}{k g}\right) \\
\varphi=\arccos \left(\frac{v_{y}-v_{0}}{k g}\right), \\
\gamma=\arccos \left(\frac{v_{z}-v_{0}}{k g}\right)
\end{array}\right.
$$

Since vx, vy and vz are known, it is important to know the output voltage from $\mathrm{x}, \mathrm{y}$, and $\mathrm{z}$ axes of the accelerometer. When we measure the output voltage, we can compute the angle of tilt $(\theta, \varphi$ and $\gamma)$ in formula (2). By analyzing the static and dynamic processes, mechanical equipment status monitoring can be arrived at.

\subsubsection{Accelerometers module hardware design}

The ADXL330 is a small, thin, low power, complete 3-axis acceleration measurement system on a single monolithic IC. It can measure the static acceleration of gravity in tilt-sensing applications, as well as dynamic acceleration resulting from motion, shock, or vibration with a minimum full-scale range of $\pm 3 g$ [6]. It contains a polysilicon surface micromachined sensor and signal conditioning circuitry to implement an open-loop acceleration measurement architecture. The output signals are analog voltages that are proportional to acceleration. The user selects the bandwidth of the accelerometer by adding the capacitors at the XOUT, YOUT, and ZOUT pins, which improves measurement resolution and helps prevent aliasing. Bandwidths can be selected to suit the application, with a range of $0.5 \mathrm{~Hz}$ to $1,600 \mathrm{~Hz}$ for $\mathrm{X}$ and $\mathrm{Y}$ axes, and a range of $0.5 \mathrm{~Hz}$ to $550 \mathrm{~Hz}$ for the $\mathrm{Z}$ axis. The accelerometer module is shown in Figure 5 . 


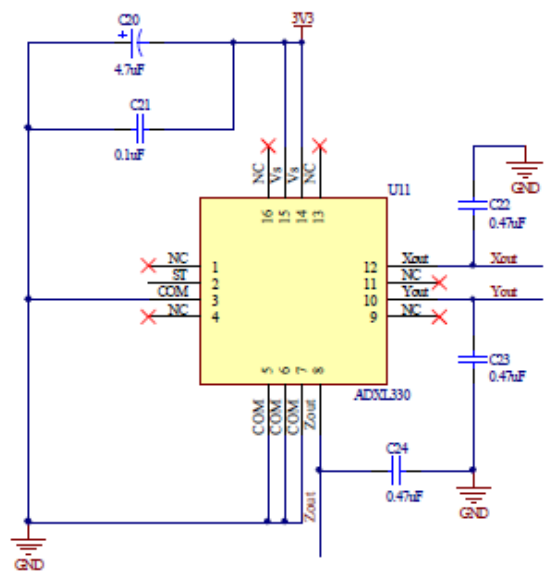

Figure 5. Accelerometer Module Circuit

\subsection{Software design}

\subsubsection{Software design of wireless module}

The software design of wireless module is based on Robert Reese's MSSTATE_IRWPAN protocol stack, who is the professor of Mississippi state university of United States. Protocol stack supports for coordinator node, router nodes and Reduced Function Device (RFD) nodes. The tree routing algorithms which can form a simple network and more complex star tree network is used in protocol stack.

The specific software features of wireless module are: Coordinator build a wireless local area acceleration sensor network, which mainly responsible for packet routing and indirect news forwarding; Once router and RFD receive acquisition data command from coordinator after router and RFD joining the corresponding wireless acceleration sensor network, it will send the acceleration value of three direction to the coordinator periodically. In order to reduce the power consumption of the system, coordinator node is in sleep status before the timing time arrives at. The RFD and router are also in sleep status before the data acquisition command arrives at. In addition, router also plays the role of data relay transmission in the whole network. Specific node software flow chart is shown in Figure 6. In order to ensure that the sending packet is received by destination node, it takes the confirmation response mechanism of APS layer in the design of the application of the wireless module. If the sender has not received the confirmation response within the prescribed time after sending the data packet, it will send again. When the failure is up to five times, the operation is invalid. In addition, to guarantee the quality of wireless sensor network, RFD and router will regularly send Ping command to parent node to determine if I am still associated. According to MSSTATE_LRWPAN protocol stack and practical application of nodes in mechanical equipment status monitoring, coordinator, router and RFDform a relatively complex tree network. The direct message transfer mode is used between coordinator and other wireless sensor node.
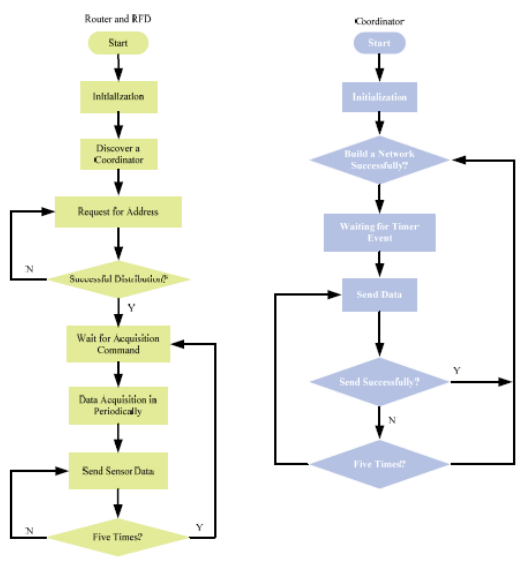

Figure 6. Flow Chart Wireless Sensor Network Node's Software

\subsection{Software design for $\mathrm{LPC} 2478$}

Design of Wireless Acceleration Sensor Nodes ... (G. VamshiKishore) 


\subsection{1. $\mu \mathrm{C} / \mathrm{OS}-\mathrm{II}$}

There are many embedded operating system can be selected for the software architecture of LPC2478 microcontroller, such as Linux, Windows CE, eCos and $\mu \mathrm{C} / \mathrm{OS}$-II. For the full functional embedded operating system (Linux, Windows CE), the software design and development will be very easy, but the embedded operating system itself will consume a lot of CPU and memory resources. These will lead to a high cost and power consumption. So, we choose $\mu \mathrm{C} / \mathrm{OS}-\mathrm{II}$. $\mu \mathrm{C} / \mathrm{OS}-\mathrm{II}$, The Real-Time Kernel, is a highly portable, ROMable, very scalable, preemptive real-time multitasking kernel RTOS for microprocessors, microcontrollers and DSPs[7]. Offering unprecedented ease-of-use, $\mu \mathrm{C} / \mathrm{OS}-\mathrm{II}$ is delivered with complete $100 \%$ ANSI C source code [8]. $\mu \mathrm{C} / \mathrm{OS}$-II manages up to 250 application tasks and provides the following services: semaphores; event flags; mutual-exclusion semaphores that eliminate unbounded priority inversions; message mailboxes and queues; task, time and timer management; and fixed sized memory block management. $\mu \mathrm{C} / \mathrm{OS}-\mathrm{II}$ can present a very small footprint (between 5 Kbytes to $24 \mathrm{Kbytes}$ ) for space-constrained embedded designs. The execution time for most services provided by $\mu \mathrm{C} / \mathrm{OS}$-II is both constant and deterministic, which do not depend on the number of tasks running in the application.

\subsection{Implementation}

We ported $\mu$ C/OS-II operating system on the NXP Semiconductors LPC2478 platform and the Embedded Operating System (EOS) supports a lot of kernel functions to handles interrupt, timer, task schedule and resources management of hardware. When the platform powered up, the initialization program, which is executable and resident in the Flash memory will, run firstly and initialize the main peripherals of the CPU, and then test the device secondly. After that some task is created, such as User Interface (UI) task, data acquisition task, data transmission task, data processing task and so on. Finally, start these tasks. The structure of software can be seen in Figure 7

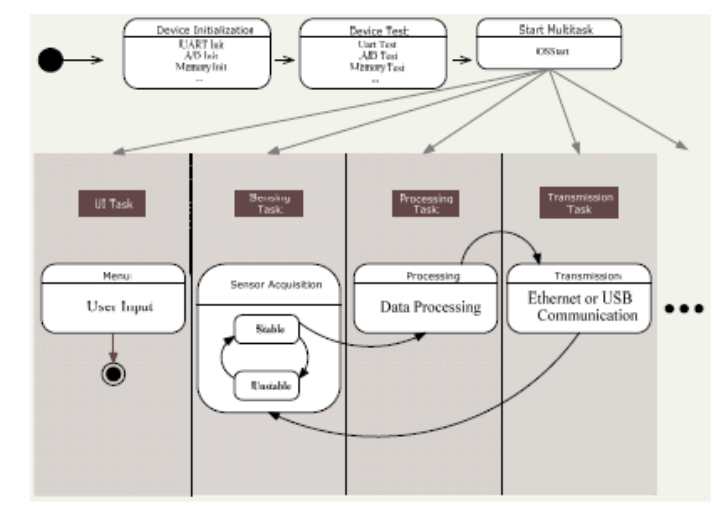

Figure 7. Firmware Based on $\mu \mathrm{c} / \mathrm{Os}-\mathrm{Ii}$ Multi-tasking Operating System

\section{EXPERIMENTS AND RESULTS}

The wireless sensor network is built with the wireless acceleration sensor nodes in the laboratory. The performance for wireless acceleration sensor node, wireless sensor network and three-axis accelerometer ADXL330 performance is tested. The whole system test platform is shown in Figure 8.

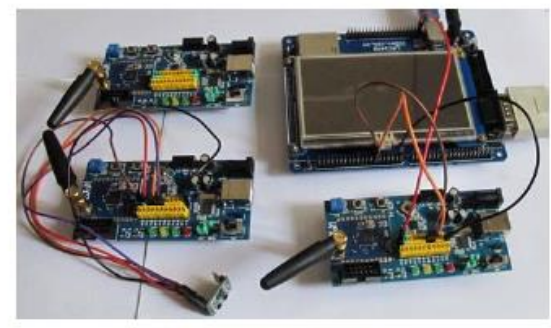

Figure 8. Testing Platform 
To test the wireless sensor network node, a wireless sensor network platform is build. The platform includes a PC, two wireless acceleration sensor nodes, and three CC2430 development board. Serial debugging assistant is used for software test. The part of test data is shown in Figure 9.

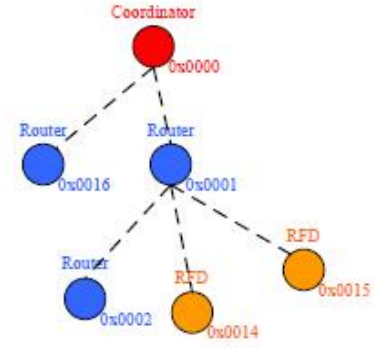

(a)

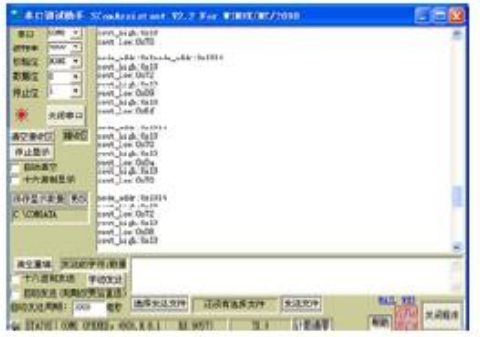

(b)

Figure 9. Network Topology of Wireless Acceleration Sensor Network, (2) the Rfd (0x0014) Data to the Router (0x0002)

To test the performance of three-axis acceleration sensor ADXL330, an experiment which is based on the three-axis accelerometer ADXL330 in the six different space positions is built. The results of CC2430 A/D channels for acceleration data are presented in table 1 .

Table 1. test data $\left(\mathrm{V}_{\mathrm{s}}=3 \mathrm{v}, \mathrm{X}_{\text {out }}=0 \mathrm{~g}, \mathrm{Y}_{\text {out }}=1 \mathrm{~g}, \mathrm{Z}_{\text {out }}=0 \mathrm{~g}\right)$

\begin{tabular}{c|c|c|c}
\hline Theory values & Samples values & Voltage values $/ \mathrm{V}$ & Practical values \\
\hline $\mathrm{X}_{\text {OUT }}=0 \mathrm{~g}$ & $0 \times 1072$ & 1.542 & $0.14 \mathrm{~g}$ \\
$\mathrm{Y}_{\text {OUT }}=1 \mathrm{~g}$ & $0 \times 1309$ & 1.785 & $0.95 \mathrm{~g}$ \\
$\mathrm{Z}_{\text {OUT }}=0 \mathrm{~g}$ & $0 \times 106 \mathrm{~F}$ & 1.541 & $0.14 \mathrm{~g}$ \\
\hline
\end{tabular}

\section{CONCLUSION}

In this paper, the design of the wireless acceleration sensor nodes used in mechanical equipment status monitoring is proposed by porting the MSSTATEI_RWPAN protocol stack on CC2430. The sensor nodes design is divided into coordinator and router (or RFD) part. Coordinator is mainly responsible for forming the root of the network tree and bridging to other networks. The task of therouter is to act as an intermediate router, passing on data from other devices. The functionality of RFD is just to talk to the parent node. These wireless sensor nodes can conveniently form a low cost, lowtransmission rate, high efficiency wireless network, which can monitor the mechanical equipment status at some test point. The experiment result shows the wireless sensor nodes and wireless sensor network performance is stable and has low packet loss rate. It can be widely used in other monitoring areas.

\section{REFERENCES}

[1] Ming Zhang, "A Novel Energy Balanced Dynamic Routing Protocol Based on Probability in Wireless Sensor Netwoks", JCIT: Journal of Convergence Information Technology, Vol. 6, No. 3, pp. 10-17, 2011.

[2] Wei LIU, Yuhua YAN, "Application of ZigBee Wireless Sensor Network in Smart Home System", IJACT : International Journal of Advancements in Computing Technology, Vol. 3, No. 5, pp. 154-160, 2011.

[3] Meiqian Ye, Tianding Chen, Changhong Yu, "ZigBee-based Positioning and Navigation System for Robot", JCIT: Journal of Convergence Information Technology, Vol. 6, No. 1, pp. 135-146, 2011.

[4] Zhaobo Liang, Yanrui Du, Runhua Liu, Bin Xie, Jin He, "Design and realization of LCD driver module based on LPC2478", Electric Power Automation Equipment, Vol. 30, No. 7, pp. 137-140, 2010.

[5] Jian HU, Haixi WU, Shuguang YE, Ling LI, Gangyan LI, "Research and Implementation of Taxi Calling-Response Method based on ZigBee", JDCTA: International Journal of Digital Content Technology and its Applications, Vol. 5, No. 8, pp. 92-100, 2011.

[6] Aner Weiss, Talia Herman, Meir Plotnik, Marina Brozgol, Inbal Maidan, Nir Giladi, Tanya Gurevich, Jeffrey M. Hausdorff, "Can an accelerometer enhance the utility of the Timed Up \& Go Test when evaluating patients with Parkinson's disease?", Medical Engineering and Physics, Vol. 32, No. 2, pp. 119-125, 2010. 